MITSUBISHI ELECTRIC RESEARCH LABORATORIES

http://www.merl.com

\title{
Parametric Compression of Rank-1 Analog Feedback in MIMO-OFDM
}

\author{
Ron Porat, Philip Orlik
}

TR2009-075 December 2009

\begin{abstract}
We consider the problem of channel state information (CSI) transmission on a fast feedback link for a multiuser-MIMO OFDM system. We compose codebook based feedback with analog based feedback and propose a new compressed analog feedback suitable for correlated antennas that provides excellent tradeoff between uplink overhead and downlink throughput.
\end{abstract}

IEEE Asilomar Conference on Signals, Systems, and Computers

This work may not be copied or reproduced in whole or in part for any commercial purpose. Permission to copy in whole or in part without payment of fee is granted for nonprofit educational and research purposes provided that all such whole or partial copies include the following: a notice that such copying is by permission of Mitsubishi Electric Research Laboratories, Inc.; an acknowledgment of the authors and individual contributions to the work; and all applicable portions of the copyright notice. Copying, reproduction, or republishing for any other purpose shall require a license with payment of fee to Mitsubishi Electric Research Laboratories, Inc. All rights reserved.

Copyright (C) Mitsubishi Electric Research Laboratories, Inc., 2009

201 Broadway, Cambridge, Massachusetts 02139 



\title{
Parametric Compression of Rank-1 Analog Feedback in MIMO-OFDM
}

\author{
Ron Porat, Phil Orlik \\ InterDigital Inc. , Mitsubishi Electric Research Labs \\ ron.porat@interdigital.com,porlik@merl.com
}

\begin{abstract}
Absract - We consider the problem of channel state information (CSI) transmission on a fast feedback link for a Multiuser-MIMO OFDM system. We compare codebook based feedback with analog based feedback and propose a new compressed analog feedback suitable for correlated antennas that provides excellent tradeoff between uplink overhead and downlink throughput.

We show via bi-directional simulation results the reliability of analog feedback in general and its advantage over codebook based feedback.
\end{abstract}

\section{INTRODUCTION}

The idea of analog feedback was proposed in the past [1] [2] in order to facilitate closed loop (CL)-MIMO in FDD systems. Here the channel can't be estimated implicitly based on uplink reference signals since down link and uplink transmissions occur in different frequency bands.

The main appeal/benefit of analog feedback is seen when it is applied to multiuser (MU)-MIMO where as was shown in [3] the channel feedback accuracy must increase linearly as the SNR increases in $\mathrm{dB}$ in order for performance to remain within a constant fixed difference from the MU-MIMO channel capacity.

Analog feedback is best suited for this task as its accuracy naturally grows with SNR.

On the other hand, the current channel feedback mechanism in next generation $4 \mathrm{G}$ cellular standards $802.16 \mathrm{~m}$ and LTE are based on codebooks [7]. By its definition, codebook operation quantizes the channel singular vectors and induces errors which will limit the performance especially for MU-MIMO.

Current $802.16 \mathrm{~m}$ and LTE systems support MU-MIMO via feedback of the channel's strongest singular vector (rank-1 feedback).

This paper compares the performance of various analog rank1 feedback mechanisms relative to codebook based feedback in typical FDD based cellular scenarios where a mobile station (MS) feeds back that information to the base station (BS) in an uplink (UL) control channel.

\section{GENERAL ANALOG RANK-1 EEDBACK}

We assume a MIMO-OFDM transmission scheme in which each transmit antenna sends an OFDM symbol consisting of $N_{\text {sub }}$, subcarriers. Our goal here is to minimize the required overhead associated with obtaining channel state information at the transmitter (CSIT). The purpose of which is to improve the reception reliability through beamforming techniques.

It is assumed that beamformed transmission will occur at the base station and thus the transmitter requires knowledge of the channel to generate its weighting vector. Mathematically, the transmitter sends the following:

$\mathbf{s}=\mathbf{w} x$

Where $\mathrm{w}$ is an Nx1 weighting vector and $x$ represents the modulation data. The optimal weighting vector is simply the singular vector associated with the largest singular value of the channel matrix $\mathbf{H}$.

In general, assuming an $\mathrm{N}$ antenna $\mathrm{BS}, \mathrm{N}$ complex valued numbers are needed to represent the strongest singular vector seen by the receiver. During the uplink transmission the mobile station (MS) can directly map the $\mathrm{N}$ complex numbers to $\mathrm{N}$ subcarriers using $\mathrm{AM}$ modulation. Additionally, repetition can be used to improve reliability at low SNR, however it is important to remember that increasing the number of BS antenna improves the UL performance and reduces the need for repetition.

A simple modification to the direct mapping makes it possible to feed back only $\mathrm{N}-1$ complex numbers by rotating all elements by the negative of the angle of the first element (thus making the first element real) and not transmitting the first element. At the BS, the first element can be computed by noting that the sum power of all elements is 1 . This scheme however makes the feedback more sensitive to power normalizations at the mobile's transmitter.

Typical feedback in $4 \mathrm{G}$ cellular systems is done per band and not per subcarrier in order to reduce uplink overhead. A typical size of band is $800 \mathrm{KHz}$ consisting of 72 contiguous subcarriers, thus we transmit a single feedback message which is some function of the channel response within the band of interest. In this case the optimal rank- 1 feedback is unknown 
but two possibilities based on averaging the channel covariance or averaging the per-subcarrrier singular vectors were shown to provide good results:

1. Compute the strongest singular vector of the average transmit covariance matrix in that band. $\hat{R}=\frac{1}{|\mathbf{S}|} \sum_{k \in \mathbf{S}} H_{k}^{H} H_{k}$ where $\mathrm{S}$ is the set of subcarriers in that band. General computation of the strongest singular vector can be facilitated in most cases using the power method [4] or via the general SVD algorithm as in [4][5]. However the complexity vastly increases for BS with 8 antennas.

2. Currently cellular mobiles have two receive antennas. In this case a simple closed form formula for the per subcarrier channel SVD for any number $\mathrm{N}$ of $\mathrm{BS}$ antennas is derived in Appendix I. Once the strongest singular vectors per subcarrier are found they can be averaged by aligning their phases with respect to any subcarrier $\mathrm{j}$ in the band

$$
v=\sum_{k \in S} v_{k} \frac{v_{k}^{*} v_{j}}{\left|v_{k}^{*} v_{j}\right|}
$$

3. Similarly, Appendix II provides an approximate closed form solution for the computation of the strongest singular vector for $4 \mathrm{xN}$ channels. MUMIMO simulations as described in section $\mathrm{V}$ using that formula show $0.5 \%$ throughput loss compared to a calculation using SVD and hence is practically perfect.

Either method above results in the generation of a representative singular vector, denoted as, $v$, for the band of interest. In the subsequent section we describe methods that can be use to further reduce the feedback overhead by using knowledge of the antenna configuration at the transmitter.

\section{PARAMETRIC COMPRESSION OF ANALOG RANK-1 FEEDBACK}

In practical cellular deployments, closely spaced antennas are likely to be used in order to reduce BS deployment cost by placing the antennas under one radome. Neighborhood restrictions and zoning laws may also require small antenna dimensions.

Other advantages of closely spaced calibrated arrays stem from the increased antenna correlation which in turn reduces the variability across frequency and time of the spatial signature of mobiles and enables improved MU-MIMO with smaller channel feedback overhead.
High correlation helps reduce the dimensionality of the rank1 feedback space and allows the compression of the feedback below $\mathrm{N}$ complex values (for $\mathrm{N}$ antenna $\mathrm{BS}$ ) by defining and feeding back only several parameters.

The following antenna configurations and feedback structures are proposed here with $\lambda$ denoting the carrier wavelength:

1. $\lambda / 2$ spaced $\mathrm{N}=2,4$ or 8 vertically polarized antennas (| or |||| or ||||||||).

In this case the strongest singular vector is approximated as a steering vector $\exp (j *(0: N-1) * \Phi) / \sqrt{8}$ and one real number $\Phi$ is fed back by using phase modulation mapping $\exp (j \Phi)$ onto one subcarrier.

2. $\lambda / 2$ spaced 2 or 4 cross polarized antennas (XX or $\mathrm{XXXX)}$ or two widely spaced clusters of antenna configuration $1 . \quad$ In this case the strongest singular vector is approximated as two steering vectors with gain and phase offset between them (applied to the first steering vector):

$$
\begin{aligned}
& {\left[r \exp (j \theta) \exp \left(j *(0: N / 2-1) * \Phi_{1}\right)\right.} \\
& \left.\exp \left(j *(0: N / 2-1) * \Phi_{2}\right)\right] / \sqrt{N\left(1+r^{2}\right) / 2}
\end{aligned}
$$

The feedback includes one complex value $r \exp (j \theta)$ which is mapped to one subcarrier ( $r=1$ on average) and two real values which are mapped to two subcarriers as in 1 .

Several other antenna configurations and compressed feedback schemes are possible but omitted here.

The parameters of the compressed feedback can be estimated in several ways:

Antenna Configuration 1 - One steering vector:

1. Denoting the optimal singular vector by $v, \Phi$ can

$$
\begin{aligned}
& \text { be estimated as } \exp (j \Phi)=\frac{x}{|x|} \text { with } \\
& x=v(2: N)^{H} * v(1: N-1)
\end{aligned}
$$

2. Using the transmit correlation matrix $\mathrm{R}$, the objective is to find a steering vector $\mathrm{V}$ that maximizes

$V^{H} R V$. Denoting the elements of the steering vector $\mathrm{V}$ by $e^{j \Phi i}$, we get 


$$
\begin{aligned}
& V^{H} R V=\sum_{m} \sum_{n} R_{m, n} e^{j \Phi(m-n)}=2 \operatorname{Re}\left\{\sum_{k=0}^{N-1} S_{k} e^{j \Phi k}\right\} \\
& \text { where } S_{k}=\sum_{n} R_{n+k, n} \text { for } \mathrm{k}>0 \text { and } \\
& S_{0}=0.5 \sum_{n} R_{n, n} .
\end{aligned}
$$

It can be clearly seen that the $\Phi$ that maximizes the above expression can be found to any degree of accuracy by taking an $\mathrm{M}$ size FFT of the $\mathrm{N}$ values $S_{k}$.

\section{Antenna Configuration Two - Two steering vectors:}

1. Using the optimal singular vector $v$ it's easy to estimate $\exp \left(j \Phi_{1}\right)=\frac{x}{|x|}$ where $x=v(2: N / 2)^{H} * v(1: N / 2-1)$ and $\exp \left(j \Phi_{2}\right)=\frac{y}{|y|}$ where $y=v(N / 2+2: N)^{H} * v(N / 2+1: N-1)$. Those estimates are then used to estimate the gain and phase offset between the two halves as

$r \exp (j \theta)=\frac{v(1: N / 2) e^{j \Phi_{1}[0: N / 2-1]^{\prime}}}{v(N / 2+1: N) e^{j \Phi_{2}[0: N / 2-1]^{\prime}}}$

2. Using the transmit covariance matrix $\mathrm{R}$ we first estimate the gain imbalance as $r=\operatorname{sqrt}\left(\frac{\sum_{i=1}^{N / 2} R_{i i}}{\sum_{i=1}^{N} R_{i i}}\right)$ and then assuming the two steering vectors are known the phase offset is estimated using the top right quadrant of $\mathrm{R}$ which represents the cross talk between the polarizations:

$$
\begin{aligned}
& R=\left[\begin{array}{ll}
Q_{1} & Q_{3} \\
Q_{3}^{H} & Q_{2}
\end{array}\right] \\
& \exp (j \theta)=\frac{X}{|x|} \text { where } \\
& X=e^{-j \Phi_{1}[0: N / 2-1]} Q_{3} e^{j \Phi_{2}[0: N / 2-1]^{\prime}} .
\end{aligned}
$$

The two steering vectors can be found separately by maximizing each one with its respective quadrant

$$
e^{-j \Phi i[0: N / 2-1]} Q_{i} e^{j \Phi_{i}[0: N / 2-1]^{\prime}} \text { as was done in the }
$$

case of one steering vector or jointly by exhaustive search maximization of $V^{H} R V$ where

$$
V=\begin{gathered}
{\left[r \exp (j \theta) \exp \left(j *(0: N / 2-1) * \Phi_{1}\right)\right.} \\
\left.\exp \left(j *(0: N / 2-1) * \Phi_{2}\right)\right] / \sqrt{N\left(1+r^{2}\right) / 2}
\end{gathered} .
$$

Separate maximization performs just slightly worse than joint maximization but can be augmented with a small joint search around the steering vectors found in the initial separate stage.

\section{ALTERNATIVE MAPPING APPROACH}

While the mapping of the parameters in this contribution is done in an analog fashion by using simple AM and PM, other mappings are possible. For example the first $n(n=2$ or more) MSB of the parameters can be sent digitally on a control channel and the difference in an analog fashion as before.

\section{SIMULATION ASSUMPTIONS}

The following MU-MIMO simulations use regularized zero forcing at the transmitter [2] whereby up to 4 users are served concurrently with each user being sent a single data stream.

Unidirectional simulations where the UL is assumed error free and bi-directional simulations with real UL channel were carried out. In bi-directional simulations the DL spectral efficiencies are computed using singular vectors estimated from an UL transmission. Thus the Unidirectional uplink error free simulations serve as an upper bound on the achievable performance.

DL spectral efficiencies are calculated assuming an MMSE receiver at the MS and assuming the interference is perfectly known at each MS.

Other simulation assumptions are as follows:

- Channel configuration is assumed Nx2 in DL and $1 \mathrm{xN}$ in $\mathrm{UL}(\mathrm{N}=4,8)$.

- DL and UL channel models use the 3GPP Spatial Channel Model (SCM) [6] Suburban and Urban Macro (15 degrees angular spread). Antenna spacing of 0.5 lambda or 4 lambda between clusters.

- $\quad$ DL Band Size - 800KHz

- DL speed and feedback delay - 3kmph, 5mS

- UL channel estimation - Real.

- CSI Feedback information - we compare with the 802.16m Rank-1 4 bit codebook (4 and 8 antennas) mapped onto 8 subcarriers via rate $1 / 4$ hamming code.

- Rank-1 Analog -

- Full information is mapped onto N subcarriers for $\mathrm{N}$ antennas

- Compressed information is mapped 
onto 1 or 3 subcarriers without repetition

- DL channel estimation - perfect

- $\quad$ DL/UL SNR per subcarrier difference - OdB. This is a result of the following assumptions:

- Total power difference between BS and MS $-23 \mathrm{~dB}$

- Power concentration ratio of 50:1 in the UL buys back $17 \mathrm{~dB}$

- UL Noise figure lower by $4 \mathrm{~dB}$

- $\quad$ UL IoT lower by $2 \mathrm{~dB}$

- User selection: 4 random users with exhaustive search

\section{SIMULATION RESULTS}

Two 8 antenna configurations were simulated. Note that as specified in section 4 , the feedback overhead was not the same for analog and codebook based methods with compressed analog feedback overhead 1 or 3 subcarriers for 8 antennas.

The antenna configuration is shown in the title of each plot and the compression algorithm used follows the description in section III.

In the legends, 'Analog' means uncompressed rank-1 analog feedback, 'Compressed Analog' uses the appropriate compression for the antenna configuration as described before and 'Perfect' means noiseless feedback channel and serves to show an upper bound of the analog feedback performance.

That upper bound can easily be achieved in most cases for the compressed analog feedback by use of repetition coding.

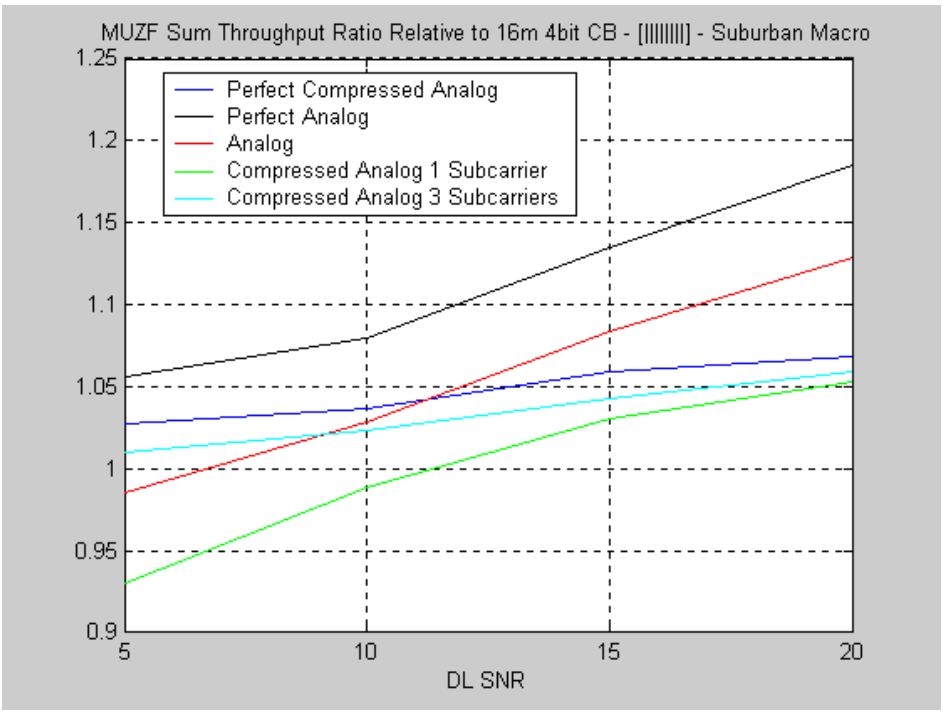

Figure 1: Eight correlated antennas throughput ratio

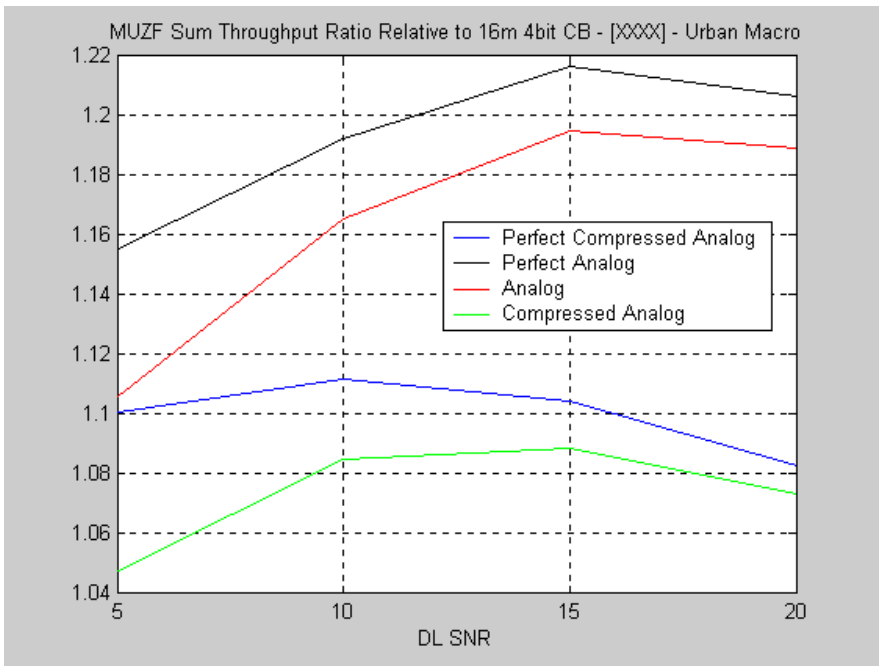

Figure 2: Four cross-polarized correlated antennas throughput ratio

\section{CONCLUSION}

It is shown that compressed rank-1 analog feedback provides excellent tradeoff of UL feedback overhead and DL MUMIMO performance and can be calculated at the MS with low complexity.

\section{ACKNOWLEDGMENT}

The authors wish to thank Yi Jiang for providing the derivation in the Appendix I. He is currently with Qualcomm Inc. and can be reached at yjiang.ee@gmail.com

\section{REFERENCES}

[1] T.L. Marzetta and B.M. Hochwald, "Fast Transfer of Channel State Information in Wireless Systems", IEEE Transactions on Signal Processing, Vol.54, No.4, April 2006.

[2] Maryam Modir Shanechi, Ron Porat and Uri Erez, “ Comparison of Practical Feedback Algorithms for Multiuser MIMO”, VTC2009 Spring

[3] N. Jindal, "MIMO Broadcast Channels with FiniteRate Feedback", IEEE Trans. Info. Theory, vol. 52, NO. 11, Nov. 2006.

[4] Golub and Van Loan "Matrix Computations"

[5] C. Studer, P. Blosch, P. Friedli, and A. Burg "Matrix Decomposition Architecture for MIMO Systems: Design and Implementation Trade-offs" Asilomar conf 2007

[6] Spatial Channel Model for MIMO Simulations http://www.3gpp.org/ftp/Specs/html-info/25996.htm

[7] IEEE $802.16 \mathrm{~m}-$ http://www.ieee802.org/16/pubs/80216m.html 


\section{APPENDIX I}

Closed Form Solution for Singular Vectors Calculation of MIMO Channels with Two Antennas at One End

Denote by $\mathrm{H}$ a $2 \mathrm{xN}$ channel. The fundamental idea is to concentrate on $H^{H}$ which is of size $\mathrm{Nx} 2$, find the right singular vectors which are of size 2 and use them to find the left singular vectors which are the right singular vectors of H.

Denote $H^{H}=V \sum U^{H}$ where $\mathrm{U}$ can be generally written as $U=\left(\begin{array}{cc}\cos \theta & \sin \theta \\ \sin \theta e^{j \phi} & -\cos \theta e^{j \phi}\end{array}\right)$

By the definition of SVD the first column of $U$ is calculated according to

$\theta, \phi=\arg \max _{\theta, \phi}\left\|H^{H}\left(\begin{array}{c}\cos \theta \\ \sin \theta e^{j \varphi}\end{array}\right)\right\| \quad$ where $\|\mathrm{x}\|$ stands for

Euclidean norm. The maximum Euclidean norm is the maximal singular value $\sigma_{1}$.

Denoting by $h_{i}$ the i'th row of $\mathrm{H}$ and developing the above expression we can derive

$e^{j \phi}=\frac{h_{2}^{*} h_{1}}{\left|h_{2}^{*} h_{1}\right|}$

Substituting we get

$\theta=\arg \max _{\theta}\left|h_{1}\right|^{2} \cos ^{2} \theta+\left|h_{2}\right|^{2} \sin ^{2} \theta+2\left|h_{2}^{*} h_{1}\right| \sin \theta \cos \theta$

Differentiating and equating to zero we can quickly find $\tan 2 \theta=\frac{2\left|h_{2}^{*} h_{1}\right|}{\left|h_{1}\right|^{2}-\left|h_{2}\right|^{2}}$ after which we use simple CORDIC rotation to calculate $\cos \theta, \sin \theta$.

Using $U H^{H}=V \sum$ we now get the singular vectors by normalizing $\quad v_{1}=h_{1}^{*} \cos \theta+h_{2}^{*} \sin \theta e^{j \phi} \quad$ and $v_{2}=h_{1}^{*} \sin \theta-h_{2}^{*} \cos \theta e^{j \phi}$

\section{APPENDIX II}

Closed Form Approximate Solution for the Strongest Singular Vector Calculation of MIMO Channels with Four Antennas at One End

The main idea is to concentrate on $H^{H}$ which is of size Nx4 and find the optimal gain and phase combining weights of the two right singular vectors of $H_{1}=H(1: 2,:)^{H}$ and $H_{2}=H(3: 4,:)^{H}$. Denote by $U_{1}$ and $U_{2}$ the two right singular vectors of the two Nx2 channels.

We would like to solve

$$
\begin{aligned}
& r, \theta=\arg \max _{r, \theta}\left\|H^{H}\left(\begin{array}{c}
r U_{1} \\
\sqrt{1-r^{2}} e^{j \theta} U_{2}
\end{array}\right)\right\|^{2}= \\
& =\arg \max _{r, \theta}\left\|\left[H_{1} H_{2}\right]\left(\begin{array}{c}
r U_{1} \\
\sqrt{1-r^{2}} e^{j \theta} U_{2}
\end{array}\right)\right\|^{2}= \\
& =r^{2}\left\|H_{1} U_{1}\right\|^{2}+\left(1-r^{2}\right)\left\|H_{2} U_{2}\right\|^{2}+ \\
& +2 r \sqrt{1-r^{2}} \operatorname{Re}\left\{e^{-j \theta}\left(H_{2} U_{2}\right)^{*}\left(H_{1} U_{1}\right)\right\}
\end{aligned}
$$

The solution for $\theta$ is straightforward and given by $\frac{x}{\|x\|}$

where $x=\left(H_{2} U_{2}\right)^{*}\left(H_{1} U_{1}\right)$

The solution for $r$ can now be found by solving $\arg \max _{r} f(r)=A r^{2}+B r \sqrt{1-r^{2}}+C$ where $A=\left\|H_{1} U_{1}\right\|^{2}-\left\|H_{2} U_{2}\right\|^{2}$ and $B=2|x|$.

Differentiating $f(r)$ and equating to zero we can arrive at a quadratic equation in $r^{2}$, the solution of which (skipping a few stages) is $r=\sqrt{0.5+\frac{A / 2}{\sqrt{A^{2}+B^{2}}}}$

Finally the rank-1 solution to $\mathrm{H}$ is given by $V=\frac{H^{H} U}{\left\|H^{H} U\right\|}$ where $U=\left(\begin{array}{c}r U_{1} \\ \sqrt{1-r^{2}} e^{j \theta} U_{2}\end{array}\right)$. 